\title{
Feasibility of high-dose dobutamine stress SSFP cine MRI at 3 Tesla with patient adaptive local RF shimming using dual-source RF transmission: initial results
}

\author{
Katharina A Strach ${ }^{1 *}$, Andreas Müller ${ }^{2}$, Marc Kouwenhoven ${ }^{3}$, Ralf Clauberg ${ }^{2}$, Claas P Naehle², Jürgen Gieseke ${ }^{4}$ \\ Hans H Schild², Daniel Thomas ${ }^{2}$
}

From 2011 SCMR/Euro CMR Joint Scientific Sessions

Nice, France. 3-6 February 2011

\section{Introduction}

Cine SSFP sequences have become the gold standard for assessment of myocardial function at $1.5 \mathrm{~T}$. However, cine imaging at higher-field strengths is hampered by SAR limitations, the increased inhomogeneity of the B1 (RF) and B0 field and the high sensitivity of SSFP sequences to off-resonance artefacts. The effect is even more pronounced in HDDS studies, which are associated with a significant increase in heart rate and consequently more complex and faster blood flow. Previously, spoiled gradient-echo sequences have been proposed as an alternative for stress studies at $3 \mathrm{~T}$. Recently, the introduction of a dual-source RF transmission system with patient-adaptive local RF-shimming has lead to a significant improvement of image quality of SSFP imaging at 3T during resting conditions and may thus allow for reliable imaging of cardiac function even at high heart rates.

\section{Purpose}

To investigate the feasibility of high-dose dobutamine stress (HDDS) imaging using SSFP sequences at 3T employing patient-adaptive local RF-shimming using a dual-source RF transmission system.

\section{Methods}

8 Patients with suspected CAD underwent a HDDS protocol on a $3 \mathrm{~T}$ MRI scanner (Achieva 3.0T-TX,

${ }^{1}$ Department of Radiology and Nuclearmedicin, University of Magdeburg, Magdeburg, Germany

Full list of author information is available at the end of the article
Philips Healthcare, Best, Netherlands), equipped with a dual-source RF transmission system. SSFP cine sequences at every stress level were performed in the short (SA), vertical (VLA) and horizontal long axis (HLA) using patient-adaptive local RF-shimming (RF$\mathrm{S})$ and compared to cine images acquired with identical sequence parameters at rest and maximal stress, without additional shimming. Overall image quality was evaluated on a 4-point grading scale (4: excellent, 1: non-diagnostic) and number of non-diagnostic segments assessed. Contrast between interventricular septum and blood pool was calculated $\left[\left(\mathrm{SI}_{\mathrm{B}}-\mathrm{SI}_{\mathrm{M}}\right) /\right.$ $\left.\left(\mathrm{SI}_{\mathrm{B}}+\mathrm{SI}_{\mathrm{M}}\right)\right]$.

\section{Results}

All HDDS studies were completed successfully. RF-S lead to a significant improvement of overall image quality at stress (SA $3.62+/-0.52$ vs. $2.13+/-0.64$; VLA 2.88 $+/-0.35$ vs. $1.75+/-0.46$; HLA $3.13+/-0.64$ vs. $1.5+/-0.76$; $\mathrm{p}<0.05$ ) and in both long axis at rest (VLA 3.38+/-0.52 vs. $2.88+/-0.35$; HLA $3.63+/-0.52$ vs. $2.75+/-0.71$; $\mathrm{p}<0.05)$ compared to sequences without RF-S. The amount of non-diagnostic segments was significantly reduced when using RF-S at rest ( 0 vs. $20, \mathrm{p}<0.05)$ and maximum stress ( 4 vs. $77, \mathrm{p} 0.05$ ) with RF-S, the difference was not statistically relevant.

\section{Conclusion}

Patient-adaptive local RF-shimming using a dual-source RF transmission MRI system allows for reliable SSFP imaging in a clinical high-dose dobutamine stress 
protocol. RF-S significantly improves image quality and reduces the number of non-diagnostic myocardial segments.

\section{Author details}

${ }^{1}$ Department of Radiology and Nuclearmedicin, University of Magdeburg, Magdeburg, Germany. ${ }^{2}$ Department of Radiology, University of Bonn, Bonn, Germany. ${ }^{3}$ Philips Healthcare, Best, Netherlands. ${ }^{4}$ Philips Healthcare,

Hamburg, Germany.

Published: 2 February 2011

doi:10.1186/1532-429X-13-S1-P101

Cite this article as: Strach et al:: Feasibility of high-dose dobutamine stress SSFP cine MRI at 3 Tesla with patient adaptive local RF shimming using dual-source RF transmission: initial results. Journal of Cardiovascular Magnetic Resonance 2011 13(Suppl 1):P101.

Submit your next manuscript to BioMed Central and take full advantage of:

- Convenient online submission

- Thorough peer review

- No space constraints or color figure charges

- Immediate publication on acceptance

- Inclusion in PubMed, CAS, Scopus and Google Scholar

- Research which is freely available for redistribution

Submit your manuscript at www.biomedcentral.com/submit 